\title{
Differentiation of mild cognitive impairment using an entorhinal cortex-based test of virtual reality navigation
}

\author{
David Howett, ',* (DAndrea Castegnaro, 2,3,* Katarzyna Krzywicka,' Johanna Hagman,' \\ Deepti Marchment,' Richard Henson, ${ }^{4}$ Miguel Rio, ${ }^{3}$ John A King, ${ }^{5}$ Neil Burgess ${ }^{2}$ and \\ Dennis Chan'
}

*These authors contributed equally to this work.

See Maass and Shine (doi:10.1093/brain/awz121) for a scientific commentary on this article.

The entorhinal cortex is one of the first regions to exhibit neurodegeneration in Alzheimer's disease, and as such identification of entorhinal cortex dysfunction may aid detection of the disease in its earliest stages. Extensive evidence demonstrates that the entorhinal cortex is critically implicated in navigation underpinned by the firing of spatially modulated neurons. This study tested the hypothesis that entorhinal-based navigation is impaired in pre-dementia Alzheimer's disease. Forty-five patients with mild cognitive impairment (26 with CSF Alzheimer's disease biomarker data: 12 biomarker-positive and 14 biomarker-negative) and 41 healthy control participants undertook an immersive virtual reality path integration test, as a measure of entorhinal-based navigation. Behavioural performance was correlated with MRI measures of entorhinal cortex volume, and the classification accuracy of the path integration task was compared with a battery of cognitive tests considered sensitive and specific for early Alzheimer's disease. Biomarker-positive patients exhibited larger errors in the navigation task than biomarker-negative patients, whose performance did not significantly differ from controls participants. Path-integration performance correlated with Alzheimer's disease molecular pathology, with levels of CSF amyloid- $\beta$ and total tau contributing independently to distance error. Path integration errors were negatively correlated with the volumes of the total entorhinal cortex and of its posteromedial subdivision. The path integration task demonstrated higher diagnostic sensitivity and specificity for differentiating biomarker positive versus negative patients (area under the curve $=0.90$ ) than was achieved by the best of the cognitive tests (area under the curve $=0.57$ ). This study demonstrates that an entorhinal cortex-based virtual reality navigation task can differentiate patients with mild cognitive impairment at low and high risk of developing dementia, with classification accuracy superior to reference cognitive tests considered to be highly sensitive to early Alzheimer's disease. This study provides evidence that navigation tasks may aid early diagnosis of Alzheimer's disease, and the basis of this in animal cellular and behavioural studies provides the opportunity to answer the unmet need for translatable outcome measures for comparing treatment effect across preclinical and clinical trial phases of future anti-Alzheimer's drugs.

1 Department of Clinical Neurosciences, University of Cambridge, Cambridge, UK

2 Institute of Cognitive Neuroscience, University College London, London, UK

3 Department of Electrical Engineering, University College London, London, UK

4 MRC Cognition and Brain Sciences Unit, and Department of Psychiatry, University of Cambridge, UK

5 Department of Clinical, Educational and Health Psychology, University College London, London, UK 
Correspondence to: Dr D. Chan

Department of Clinical Neurosciences, Herchel Smith Building, University of Cambridge

Forvie Site, Robinson Way, Cambridge, CB2 0SZ, UK

E-mail: dc598@cam.ac.uk

Keywords: Alzheimer's disease; mild cognitive impairment; entorhinal cortex; path integration; virtual reality

Abbreviations: 4MT = Four Mountains Task; ACE-R = Addenbrookes Cognitive Examination-Revised; al $/ \mathrm{m} / \mathrm{pmEC}=$ anteriorlateral/medial/posteromedial entorhinal cortex; $\mathrm{AUC}=$ area under the curve; $\mathrm{iVR}=$ immersive virtual reality; $\mathrm{MCI}(+/-)=$ mild cognitive impairment (with/without CSF evidence of underlying Alzheimer's disease); PCC = posterior cingulate cortex

\section{Introduction}

To date, all interventional trials aimed at slowing the progression of Alzheimer's disease have failed. Two of the main contributors to this failure are: (i) problems in identifying the initial stages of Alzheimer's disease, such that interventional trials are applied too late in the disease process; and (ii) the lack of translatable outcome measures for comparing treatment effects across preclinical testing in animal models of disease and clinical trials in patient populations (Mehta et al., 2017).

Detection of Alzheimer's disease-related changes in entorhinal cortex (EC) function provides a potential solution to both of these problems. Degeneration of the EC is a critical initial stage of typical Alzheimer's disease (Braak and Del Tredici, 2015), with 60\% loss of layer II EC neurons observed by the time cognitive impairment is manifest (Gomez-Isla et al., 1997). Additionally, there is emerging evidence that the initial stages of Alzheimer's disease may be associated with the trans-neuronal spread of pathological tau within the EC-hippocampal circuit (de Calignon et al., 2012; Ahmed et al., 2014), prior to neocortical infiltration. As such, tests sensitive to EC function might have added value in identifying the very earliest stages of Alzheimer's disease, prior to hippocampal involvement.

Extensive evidence from animal studies indicates that the $\mathrm{EC}$ is involved in spatial navigation. In vivo single cell studies have identified EC neurons with spatially-modulated firing patterns, including grid cells (Hafting et al., 2005), head direction cells (Sargolini et al., 2006) and border cells (Solstad et al., 2008), with firing activity coupled to spatial behaviours (McNaughton et al., 2006). Together with hippocampal place cells (O'Keefe and Dostrovsky, 1971), these EC cells are considered to represent the neural basis of a cognitive map (O'Keefe and Nadel, 1978) that mediates spatial behaviours (Fyhn et al., 2007; Lester et al., 2017). Within the EC, the medial EC (mEC) is considered to be particularly involved in navigation, given that up to $95 \%$ of mEC neurons may be grid cells (Diehl et al., 2017), in contrast to lateral EC neurons, which exhibit little spatial selectivity. Evidence that the EC underpins navigation in other mammalian species is supported by the demonstration of EC grid cells in bats (Yartsev et al., 2011), monkeys (Killian et al., 2012) and humans (Jacobs et al., 2013).
The EC is a phylogenetically conserved structure with distinct medial and lateral subdivisions that are homologous with the anterolateral EC (alEC) and posteromedial EC (pmEC) in humans (Navarro Schröder et al., 2015; Naumann et al., 2016, 2018). Sensory characteristics of objects appear to be represented in the alEC (Olsen et al., 2017; Yeung et al., 2017; Reagh et al., 2018) while representations of scenes and current location based upon selfmotion are represented in the pmEC (Reagh and Yassa, 2014; Berron et al., 2018; Campbell et al., 2018), with both streams converging on the hippocampus. Thus, integration of self-motion cues requires the pmEC, whereas remembering the spatial configuration of an environment and the objects/contents within it is dependent upon the hippocampus (Knierim et al., 2014). Path integration is the ability to keep track of, and return to, a previously visited location and is dependent upon the continuous integration of multisensory cues (visual, proprioceptive and vestibular) representing current position and heading direction in reference to a fixed location (Etienne and Jeffery, 2004; McNaughton et al., 2006). While several brain regions are thought to contribute to path integration there is robust evidence that the EC and the periodic firing of grid cells are central to this navigation strategy (McNaughton et al., 2006; Burgess, 2008), supplemented by inputs from other EC cells with spatially-modulated firing, notably head direction cells. Moreover in rodents, path integration deficits are elicited by mEC lesions (Parron and Save, 2004; Van Cauter et al., 2013; Knierim et al., 2014; Jacob et al., 2017) and attenuated grid cell firing achieved via both mEC layer 2 knockout (Tennant et al., 2018), and glutamatergic receptor 1 knockout (Allen et al., 2014). This evidence from the animal literature is reinforced by human imaging studies that demonstrate the role of the EC in components of path integration such as route planning (Maguire et al., 1998; Jacobs et al., 2010), computation of goal direction (Chadwick et al., 2015) and goal distance (Spiers and Maguire, 2007; Howard et al., 2014). Finally, dysfunction of grid cell-like activity is associated with path integration deficits in older adults (Stangl et al., 2018).

Spatial tests, based on the cognitive map theory, have already shown that spatial processing is impaired in early Alzheimer's disease. The Four Mountains Test (4MT), a hippocampal-dependent test of allocentric spatial memory (Hartley et al., 2007), differentiates patients with mild cognitive impairment (MCI) with and without CSF biomarkers of 
Alzheimer's disease (MCI+ and $\mathrm{MCI}-$, respectively) (Moodley et al., 2015) and is predictive of conversion from MCI to dementia (Wood et al., 2016). Crucially for detection of Alzheimer's disease prior to symptom onset, performance on the 4MT correlates with dementia risk score in asymptomatic 40-59 year olds (Ritchie et al., 2018), while young adult $A P O E \& 4$ carriers at increased risk of Alzheimer's disease exhibit reduced grid-cell like representations and activation of the EC during a functional MRI navigation task (Kunz et al., 2015). Finally, impaired route learning and way-finding is observed in asymptomatic individuals with positive amyloid-PET scans (Allison et al., 2016).

These previous studies provide the backdrop for the present study, which investigates EC-based navigation in MCI patients at risk of developing dementia. Navigation will be tested using a path integration task (Fig. 1A). In this study, path integration will be tested using an immersive virtual reality (iVR) paradigm where participants navigate by realworld walking within simulated environments. Immersive VR has several theoretical and operational advantages over 'desktop' VR tasks, which are typically performed seated and thus without locomotor or proprioceptive feedback, both of which are pivotal for grid cell function (Winter et al., 2015). First, the actual movement in iVR approximates real world navigation and thus has greater ecological validity than desktop VR. Second, there is evidence of differing neural processes underlying desktop and actual navigation, with desktop VR being associated with lower frequency hippocampal theta oscillations (Bohbot et al., 2017). This has negative implications for desktop VR as a valid proxy for real life navigation. Lastly, larger rotational (Klatzky et al., 1998) and distance errors (Distler et al., 1998; Sinai et al., 1999; Adamo et al., 2012) have been reported in desktop VR navigation when compared with tasks requiring active movement, possibly reflecting the absence of self-motion cues, leading in turn to reduced grid cell activation (Ólafsdóttir and Barry, 2015).

The primary objective of this study was to test the hypothesis that performance on an iVR path integration task of EC function would differentiate MCI patients at increased risk of developing dementia. The secondary study objectives were to determine: (i) whether manipulation of the environmental conditions would affect path integration performance; (ii) whether path integration test performance correlates with EC volumes; and (iii) whether the path integration task exhibits greater classification accuracy than current cognitive tests considered to have high diagnostic sensitivity and specificity for early Alzheimer's disease.

\section{Materials and methods}

\section{Participants}

Patients with MCI $(n=45)$ were recruited from the Cambridge University Hospitals NHS Trust Mild Cognitive Impairment and Memory Clinics. MCI was diagnosed by neurologists according to the Petersen criteria (Petersen, 2004), diagnosis of which requires: (i) subjective cognitive complaint; (ii) objective evidence of cognitive impairment; (iii) preserved activities of daily living; (iv) functional independence; and (v) absence of dementia. Objective cognitive decline was evaluated using the Addenbrooke's Cognitive Examination-Revised (ACE-R; Mioshi et al., 2006) and a score of 0.5 on the Clinical Dementia Rating scale (CDR) (Morris, 1997). All patients underwent screening blood tests to exclude reversible causes of cognitive impairment. Exclusion criteria included the presence of a major medical or psychiatric disorder, epilepsy, a Hachinski Ischaemic Score $>4$ (Moroney et al., 1997), a history of alcohol excess or any visual or mobility impairment of such severity as to compromise ability to undertake the iVR test.

Twenty-six patients with MCI underwent CSF biomarker studies (amyloid- $\beta_{1-42}$, total tau, phosphorylated tau) as part of their clinical diagnostic workup. Biomarker studies were undertaken using ELISA assay kits (Innotest, Innogenetics) as outlined elsewhere (Shaw et al., 2009). Thresholds for positivity were set as CSF amyloid $<550 \mathrm{pg} / \mathrm{ml}$, CSF tau $>375 \mathrm{pg} / \mathrm{ml}$ with a CSF tau: amyloid ratio of $>0.8$ (Mulder et al., 2010). MCI patients were stratified into biomarker-positive $(\mathrm{MCI}+$, $n=12)$ and biomarker-negative (MCI-, $n=14$ ) groups (Table 1). Researchers undertaking the VR tests were blinded to the CSF status of patients. The remaining 19 patients with MCI did not undergo CSF studies as part of their clinical workup. Healthy control participants without a history of cognitive impairment (healthy control subjects, $n=41$, Table 1) were recruited from Join Dementia Research, an online repository of patients and volunteers interested in participating in dementia research.

The study was undertaken in line with the regulations outlined in the Declaration of Helsinki (WMA, 2013) and was approved by the NHS Cambridge South Research Ethics Committee (REC reference: 16/EE/0215).

\section{The immersive virtual reality path integration task}

The path integration task was administered using the HTC Vive iVR kit (Fig. 1B), which uses external base stations to map out a $3.5 \times 3.5 \mathrm{~m}$ space within which participants walked during the VR task. If participants went beyond the tracked boundary by $30 \mathrm{~cm}$, an 'out of border' warning appeared in their sightline to encourage them to not walk any further. Researchers were also in the immediate proximity to ensure that participants did not venture beyond the test space.

The task was programmed in the Unity game engine and ran on Steam VR software, running on an MSI VR One backpack laptop.

The path integration task was undertaken within virtual open arena environments with boundary cues projected to infinity (Fig. 1C). Three environments were used, each with unique surface details, boundary cues and lighting. The absence of local landmarks ensured EC-grid cell dependent strategies rather than striatal-mediated landmark-based navigation (Doeller et al., 2008).

No enclosure or local landmarks were present during the task in order to exclude any non-path integration compensatory navigation strategies. A 1:1 correspondence between movement in the real and virtual worlds eliminated vestibular mismatch and minimized nausea and other tolerability issues. 
A

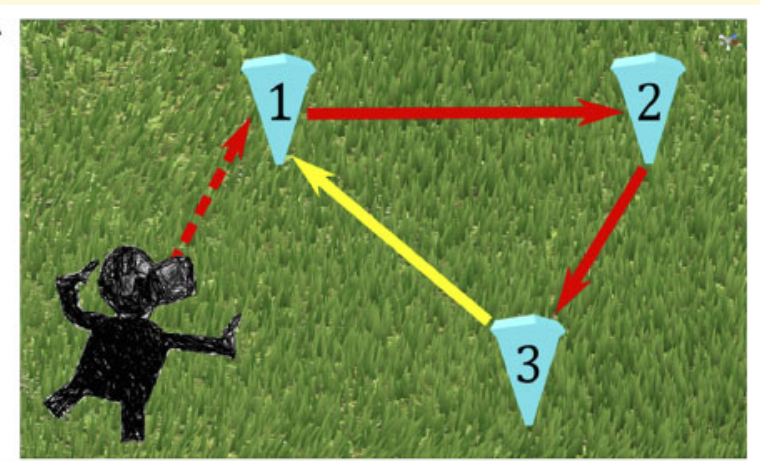

C

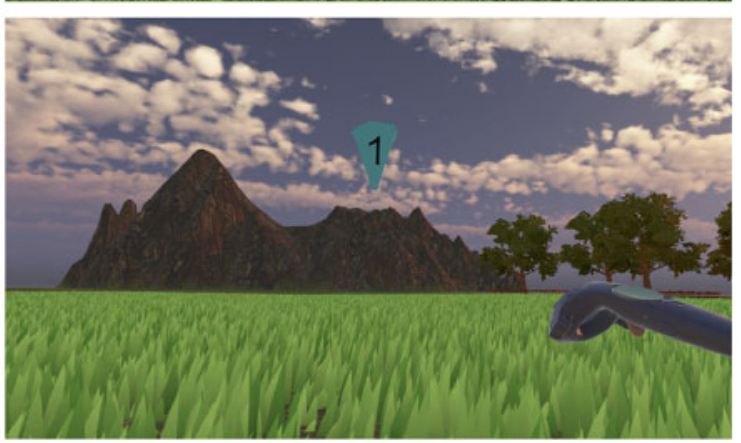

E

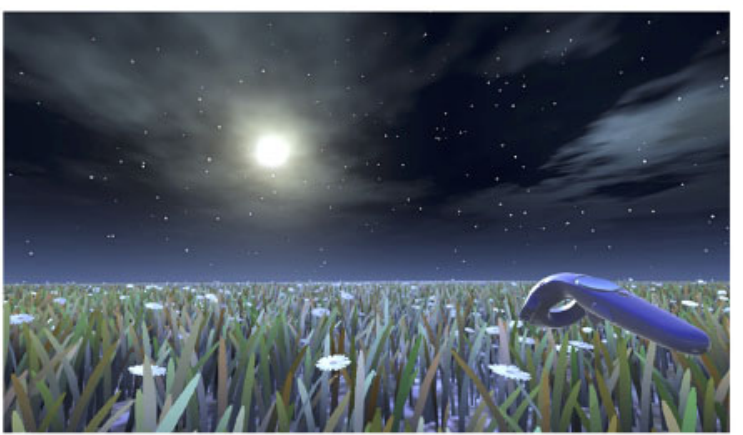

B

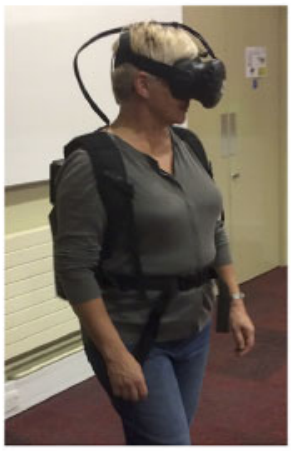

D

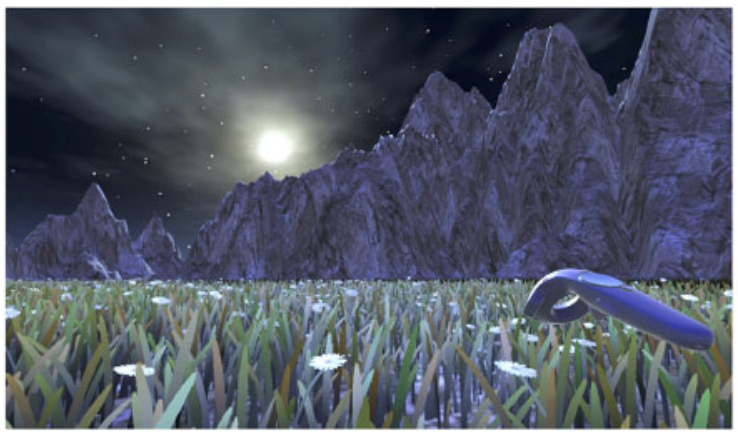

F

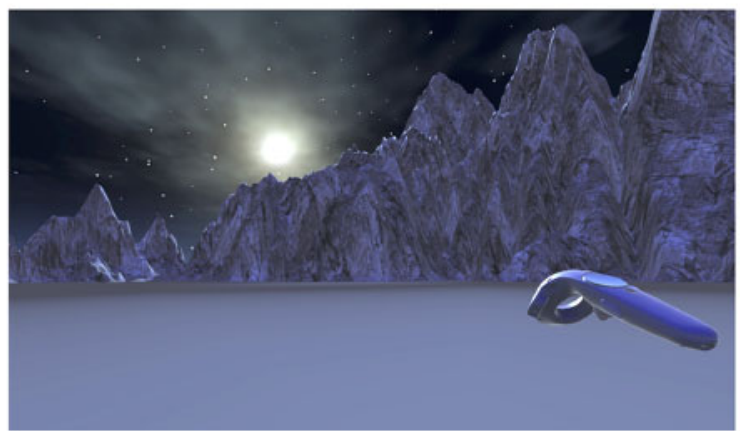

Figure I Path integration task. (A) Illustration of the path integration task. Each numbered inverted blue cone is a location marker. Only one cone was visible at a time; upon reaching a blue cone it disappeared and the next one in the sequence appeared. Red arrows indicate the guided sequence along two sides of the triangle. The yellow arrow, the last side of the triangle, signifies the assessed return path, performed in the absence of any cones. (B) Demonstration of VR equipment on a participant during the task, used with permission. (C) Example environment from the head mounted display with textural and boundary cues present, with cone I and the controller shown. Texture and boundary cues are present in all trials when navigating between cones. (D-F) Return conditions applied when attempting to return to the location of cone I only (yellow arrow, $\mathbf{A}$ ) and included no change (D), removal of environment boundaries (E) and removal of surface detail (F).

Participants were asked to walk an 'L'-shaped outward path to three different locations, each marked by inverted cones at head height numbered one, two and three (Fig. 1A and C). Only one cone was present at a time, each cone would disappear after the participant reached it and the next cone in sequence would appear. An auditory stimulus was presented with a cone's appearance prompting participants toward the next cone's location. Upon reaching cone 3, a message projected onto the virtual scene asking participants to walk back to their remembered location of cone 1 (return path). When they reached their estimated location of cone 1, participants pressed a trigger on a hand-held controller that logged their location and ended the trial.

Pre-trial practice sessions consisted of $20 \mathrm{~s}$ of habituation to the iVR environment, during which participants were encouraged to explore the environment. Following habituation, participants performed five practice trials, where cone
1 was re-presented at the end of each trial to provide direct visual feedback to participants on the distance error between the remembered and actual locations of cone 1 .

The task consisted of nine trials conducted within each of the three environments, totalling 27 trials per participant. To examine the effects of environmental cues on path integration, the environment was altered during the return path when participants were attempting to return to the remembered location of cone 1 . Three return conditions were used: condition A, no environmental change (Fig. 1D); condition B, removal of boundary cues (Fig. 1E); and condition C, removal of surface detail (Fig. 1F).

Each return condition was presented three times per environment, with return conditions presented pseudo-randomly in each environment in order to ensure participants were relying more on proprioceptive and self-motion cues rather than allothetic strategies. 
Table I Demographics and neuropsychological test scores of across patients with mild cognitive impairment and healthy controls participants

\begin{tabular}{|c|c|c|c|c|}
\hline & \multirow{2}{*}{$\begin{array}{l}\text { Healthy } \\
\text { controls }(n=4 I)\end{array}$} & \multicolumn{3}{|l|}{$\mathrm{MCl}(n=45)$} \\
\hline & & $\operatorname{MCl}(n=45)$ & Negative $(n=14)$ & Positive $(n=12)$ \\
\hline Age & $69.3 \pm 7.5$ & $71.7 \pm 8.3^{n . s}$ & $71.1 \pm 9.0$ & $75.4 \pm 7.0^{\mathrm{n} . \mathrm{s}}$ \\
\hline Males (\%) & $15(36)$ & $12(63)^{\mathrm{n} . \mathrm{s}}$ & $10(7 I)$ & $9(75)^{\text {n.s }}$ \\
\hline Years in education & $|4.8 \pm 3.6|$ & $14.2 \pm 3.37^{\text {n.s }}$ & $14.5 \pm 4.4$ & $14.5 \pm 3.8^{\mathrm{n} . \mathrm{s}}$ \\
\hline ACE-R & $97.2 \pm 3.2$ & $89.3 \pm 5.4^{*}$ & $86.6 \pm 7.6$ & $80.1 \pm 12.1^{n . s}$ \\
\hline MMSE & $29.7 \pm 0.6$ & $27.90 \pm 1.7^{*}$ & $27.6 \pm 2.6$ & $25.0 \pm 1.7^{\mathrm{n} . \mathrm{s}}$ \\
\hline NART Errors & $6.28 \pm 3.40$ & $17 \pm 10.95^{*}$ & $13.1 \pm 8.9$ & $9.1 \pm 6.8^{\mathrm{n} . \mathrm{s}}$ \\
\hline \multicolumn{5}{|l|}{ Rey Figure Recall } \\
\hline Copy & $36 \pm 0$ & $34.2 \pm 2.7^{*}$ & $34.4 \pm 1.7$ & $33.1 \pm 4.4^{\mathrm{n} . \mathrm{s}}$ \\
\hline Immediate & $22.2 \pm 7.6$ & $17.5 \pm 9.8^{*}$ & $13.8 \pm 8.3$ & $9.6 \pm 9.1^{\text {n.s }}$ \\
\hline Delayed & $21.3 \pm 7.9$ & $15.8 \pm 11.0 *$ & $12.8 \pm 9.8$ & $8.3 \pm 9.6^{\mathrm{n} . \mathrm{s}}$ \\
\hline \multicolumn{5}{|l|}{ FCSRT immediate } \\
\hline Free & $34.3 \pm 5.1$ & $24.9 \pm 11.5^{*}$ & $22.1 \pm 9.2$ & $|5.4 \pm 1| .4^{*}$ \\
\hline Total & $47.6 \pm 0.6$ & $44.7 \pm 5.7^{*}$ & $43.1 \pm 8.3$ & $36.1 \pm 11.5^{\mathrm{n} . \mathrm{s}}$ \\
\hline \multicolumn{5}{|l|}{ FCSRT delayed } \\
\hline Free & $13.4 \pm 1.5$ & $9.3 \pm 5.3^{*}$ & $7.9 \pm 5.1$ & $4.8 \pm 4.8^{\mathrm{n} . \mathrm{s}}$ \\
\hline Total & $16 \pm 0$ & $14.8 \pm 2.3^{*}$ & $13.9 \pm 4.1$ & $12.3 \pm 4.0^{\mathrm{n} . \mathrm{s}}$ \\
\hline Trails B, s & $77.2 \pm 26.5$ & $145.6 \pm 72.8^{*}$ & $130.1 \pm 42.2$ & $152.6 \pm 88.6^{\mathrm{n} . \mathrm{s}}$ \\
\hline Digit Symbol & $64.2 \pm 14.5$ & $49.9 \pm 14 . I^{*}$ & $47.0 \pm 7.5$ & $43.7 \pm 13.7^{\mathrm{n} . \mathrm{s}}$ \\
\hline $4 \mathrm{MT}$ & $10.8 \pm 1.8$ & $9.3 \pm 3.0^{*}$ & $7.3 \pm 3.4$ & $6.8 \pm 2.2^{\mathrm{n} . \mathrm{s}}$ \\
\hline
\end{tabular}

Between group differences in neuropsychological test performance were assessed between healthy control subjects versus $\mathrm{MCl}$ as a whole, and $\mathrm{MCl}+$ versus $\mathrm{MCl}-$, scores indicate number of correct responses unless otherwise indicated. $* P<0.05, * P<0.02$ (Bonferroni-adjusted alpha); n.s $=P>0.05$.

Digit Symbol = Digit Symbol Substitution Test; FCSRT = Free and Cued Selective Reminding Test; MMSE = Mini-Mental State Examination; NART = National Adult Reading Test; Trails $B=$ Trail Making Test $B$.

Condition B was designed to increase dependence on selfmotion cues and homing vector calculation by removing boundary cue information (Burgess et al., 2004), thereby placing a greater cognitive load on the path integration network (Zhao and Warren, 2015). Condition C was designed to prevent feedback from surface motion during locomotion, thereby disrupting optic flow (Kearns et al., 2002) and increasing dependence on allocentric representations of space (Nardini et al., 2008). As such, return conditions $\mathrm{B}$ and $\mathrm{C}$ were considered analogous to 'stress tests' for EC network-dependent navigation, with the prediction that a greater impairment in task performance would be observed during these conditions, compared with condition A.

Performance in the iVR path integration task was assessed using three outcome measures. Absolute distance error, the primary outcome measure, was defined as the Euclidean distance between estimated and actual location of cone 1, in line with previous research (Fig. 2; Chrastil et al., 2015; Mokrisova et al., 2016). Two secondary outcome measures were included to deconstruct absolute distance errors into its proportional angular and linear components. These measures additionally controlled for between-trial variance in triangle geometry owing to the pseudo-random generation of cone locations that could affect task difficulty, although variance is minimal in paths $<10 \mathrm{~m}$ long (Harris and Wolbers, 2012). Proportional angular errors reflect the accuracy of performed rotation at cone 3 toward the participant's estimated location of cone 1 compared to the optimal

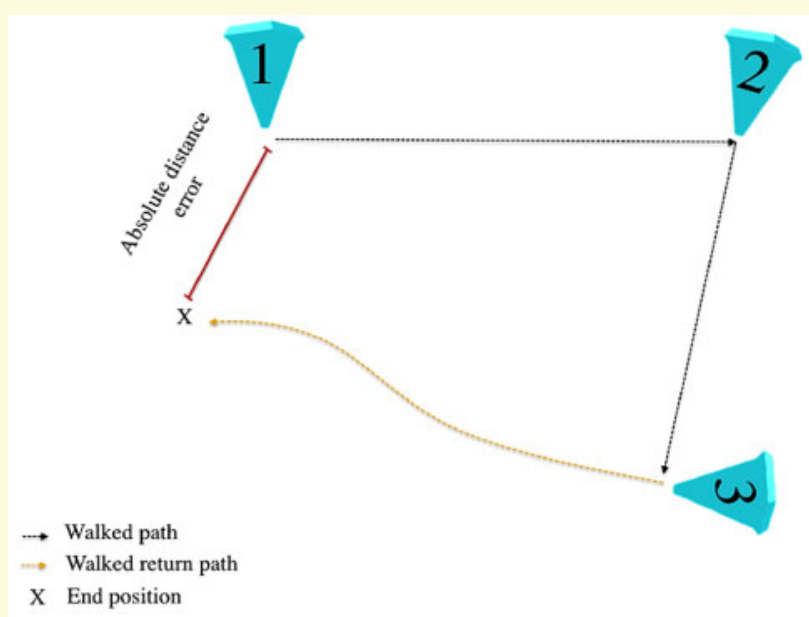

Figure 2 Primary measures of performance accuracy. Absolute distance error is defined as the Euclidean distance between the participant's estimate of location one (goal) and the actual location of cone I.

rotation required to align with cone 1 (Supplementary Fig. 1A). Proportional linear errors reflected the accuracy of distance estimation, with the Euclidean distance travelled between cone 3 and the participant's estimated location of cone 1 compared to the distance between cone 3 and actual location of cone 1 (Supplementary Fig. 1B). 


\section{MRI acquisition and analysis}

Thirty-seven healthy control subjects and 34 MCI patients (11 $\mathrm{MCI}+, 9 \mathrm{MCI}-$ ) underwent MRI scanning on 32 channel Siemens $3 \mathrm{~T}$ Prisma scanners based either at the MRC Cognition and Brain Sciences Unit, Cambridge, or the Wolfson Brain Imaging Centre, Cambridge, with the same acquisition parameters used at the two scan sites. The scan protocol included whole brain $1 \times 1 \times 1 \mathrm{~mm} \mathrm{~T}_{1}$-weighted MPRAGE (acquisition time $5 \mathrm{~min} 12 \mathrm{~s}$, repetition time $2300 \mathrm{~ms}$, echo time $2.96 \mathrm{~ms}$ ) and high-resolution $0.4 \times 0.4 \times 2 \mathrm{~mm} \mathrm{~T}_{2}$-weighted scans through the hippocampal formation with scans aligned orthogonally to the long axis of the hippocampus (acquisition time $8 \mathrm{~min} 11 \mathrm{~s}$, repetition time $8020 \mathrm{~ms}$, echo time $50 \mathrm{~ms}$ ).

As well as segmentation of the whole EC, additional segmentation of the alEC and pmEC subregions, representing the human homologues of the rodent lateral and medial EC, respectively, was undertaken given their differing roles in spatial processing (Van Cauter et al., 2013; Knierim et al., 2014). Converging evidence from functional MRI studies indicate a functional domain-specificity to the EC that mirrors rodent research, whereby the pmEC and alEC is implicated in processing scene and object information, respectively (Maass et al., 2015; Navarro Schröder et al., 2015). Whilst age-related deficits in object-related processing have been related to alEC hypoactivity (Berron et al., 2018; Reagh et al., 2018), no research to date has investigated the relationship between EC subfield volumetry and performance in navigational tasks in either MCI or Alzheimer's disease. While segmentation protocols for these subregions are available at $7 \mathrm{~T}$ (Maass et al., 2015), no complete protocol is available for segmentation at $3 \mathrm{~T}$. Therefore, for this study, an in-house protocol was devised that partially segmented alEC and pmEC using the three anterior-most and three posterior-most slices of the EC. Intermediate slices were not used for alEC and pmEC segmentation because of the overlap of the two subdivisions within this part of the EC and the absence of robust anatomical landmarks for delineating this progressive boundary. As such, this protocol prioritized specificity of segmentation over completeness (Supplementary material). Manual segmentation was performed in ITK-SNAP (Yushkevich et al., 2006) (Fig. 3, detailed protocol in Supplementary material). High inter- and intrarater reliability was achieved for the manual segmentation protocol of the EC, alEC and pmEC, consistent with previous research (Berron et al., 2017; Olsen et al., 2017) (Supplementary Table 1).

Given the involvement of the hippocampus and retrosplenial cortex in path integration (Worsley et al., 2001; Chrastil et al., 2015 ) these additional regions of interest were also segmented using Freesurfer 6.0 (Fischl et al., 2002; Iglesias et al., 2015). In the absence of an automated protocol for the complete segmentation of the retrosplenial cortex, posterior cingulate cortex (PCC) and isthmus of cingulate cortex masks were used as a proxy measure of the retrosplenial cortex, with the former encompasses the retrosplenial cortex along with other structures and the latter targeting the ventral retrosplenial cortex implicated in processing scene information (Vann et al., 2009; Mitchell et al., 2018).

All segmentations were manually inspected to exclude cysts, CSF and meninges; all volumetric measurements were averaged between hemispheres and normalized to intracranial volume.

\section{Comparator neuropsychological tests}

To compare the ability of the iVR test to classify prodromal Alzheimer's disease with that of reference neuropsychological tests considered to be highly sensitive to early Alzheimer's disease. All participants were administered a battery of tests chosen for their effectiveness in predicting conversion from MCI to dementia (i-iii), inclusion in the Preclinical Alzheimer's Cognitive Composite (i and iv) approved by the FDA for use as cognitive outcome measures in trials aimed at preclinical Alzheimer's disease, or prior work indicating high sensitivity and specificity for prodromal Alzheimer's disease (v).

These tests are as follows (cognitive domains assessed in parentheses): (i) Free and Cued Selective Reminding Test (FCSRT, episodic memory - verbal; Buschke, 1984); (ii) Rey figure recall (RFR, episodic memory - non-verbal, Osterrieth, 1944); (iii) Trail Making Test B (TMT-B; executive function, attention, processing speed; Bowie and Harvey, 2006); (iv) Digit Symbol test (DST, attention, processing speed, Ryan and Lopez, 2001); and (v) 4MT (allocentric spatial memory; Hartley et al., 2007). All participants also underwent global cognitive testing with the ACE-R (Mioshi et al., 2006) and the National Adult Reading Test (NART; Nelson, 1982), as a measure of premorbid IQ.

\section{Statistical analysis}

Demographic differences between $\mathrm{MCI}+$ and $\mathrm{MCI}-$ were assessed using one-way ANOVA, or the Kruskal Wallis test where parametric assumptions were violated, whereas differences between healthy control subjects and total (combined) MCI were assessed using $t$-tests or non-parametric MannWhitney test.

Between-group performance in the path integration task compared all MCIs against healthy control subjects, as well as MCI + against MCI-. Linear mixed effect modelling (LME) was used to assess the effect of MCI status on absolute distance error, proportional angular error and proportional linear error. LMEs are the most suitable method for analysing clustered datasets (27 trials with one of three return conditions per trial per participant), with missing data (excluded due to travelling 'out of border', see 'Results' section), and unbalanced designs (Moen et al., 2016). Final model fixed effects included an interaction term between diagnosis and return condition, along with covariates of age, sex, years in education, ACE-R, NART and VR environment. Unique participant identifiers were used as the random intercept and VR environment as random coefficient, for further details see the Supplementary material. Reported denominator degrees of freedom were computed using the conservative Satterthwaite approximation.

Between-group differences in region of interest volumetry and cognitive performance across the neuropsychological test battery were investigated using one-way ANCOVA-rank ordered where parametric assumptions were violated (Conover and Iman, 1982)—covarying for age, sex and years in education. Separate linear regression models were used to assess absolute distance error (averaged per participant across all trials) and region of interest volumetry, adjusting for age, sex, years in education and mean path integration performance per participant group. Analyses were conducted across all 


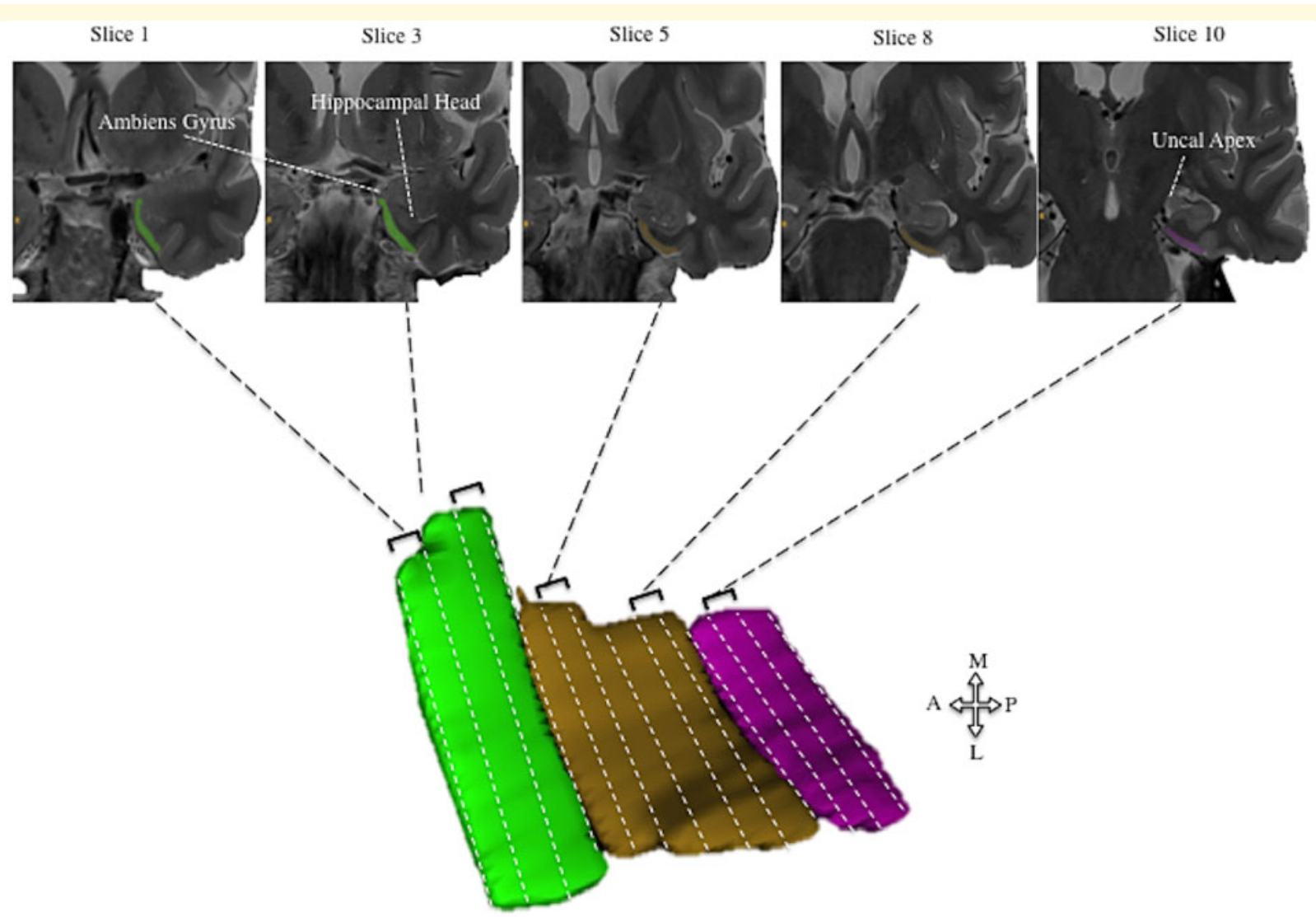

Figure 3 Protocol for the segmentation of the whole entorhinal cortex and partial segmentation of its anteriolateral and posterioromedial subdivisions. Anteriolateral EC (alEC, green) is segmented two slices anterior to the emergence of the hippocampal head (slice 3). Posteromedial (pmEC, pink) is segmented from one slice anterior to, and one slice posterior from, the uncal apex (slice I0). This partial approach for EC subdivisions does not encompasses the full extent of the EC but rather reflects the anterior and posterior extremes of the EC that avoid segmenting the progressive boundary in the absence of consistent landmarks. Correspondingly, all intermediate slices between alEC and pmEC are segmented as EC (brown), total EC volume is produced by summing all three EC subdivision volumes. Arrow schematic indicates anatomical plane for 3D segmentation.

participants and between $\mathrm{MCI}+$ versus $\mathrm{MCI}-$. Bonferroni correction was used to control for planned multiple comparisons. Residuals were visually inspected for violating linear assumptions, leverage and outliers.

The classification ability (MCI from healthy control subjects or $\mathrm{MCI}+$ from $\mathrm{MCI}-$ ) of the path integration test (using per-participant averages of each path integration outcome measure) was compared to reference cognitive tests. All linear classification models were adjusted for age, sex and years in education and used $\mathrm{k}$-fold cross-validation $(\mathrm{k}=10)$ to control for over-fitting (Hawkins et al., 2003). Posterior probabilities following cross-validation were used to generate area under the curve (AUC) of the receiver operating characteristic (ROC), as well as optimal sensitivity and specificity. Pointwise confidence intervals were generated following bootstrapping with 1000 replications.

All analysis was conducted in MATLAB 2017b (Mathworks, https://uk.mathworks.com/).

\section{Data availability}

Anonymized data are available on request.

\section{Results}

\section{Demographics and neuropsychological testing}

No significant differences in age, gender, or years in education were observed between all MCI and healthy control subjects, or between MCI + and MCI- (Table 1). Following Bonferroni correction with an adjusted $\alpha$ of 0.002 , the MCI group as a whole exhibited significantly more errors in all neuropsychological tests compared to healthy control subjects $(P<0.002)$, whereas no difference between $\mathrm{MCI}+$ and $\mathrm{MCI}-$ survived multiple comparisons $(P>0.002)$.

\section{Immersive virtual reality path integration task}

Of 2295 trials, 775 were excluded $(33.77 \%)$ because of the 'out of border' boundary being reached during the return 
path, leaving 1520 viable trial remaining for analysis, with no between group difference in 'out of border' warnings were observed $(P>0.05)$. All participants successfully completed the path integration task with no reported nausea or tolerability issues.

\section{Absolute distance error}

The MCI group as a whole exhibited significantly larger absolute distance errors than the healthy control group $[t(1,107)=3.24, P<0.01$, Fig. $4 \mathrm{~A}]$, with an estimated $57.33 \pm 17.87 \mathrm{~cm}$ increase in absolute distance error compared to healthy control subjects. MCI + patients exhibited significantly larger absolute distance errors compared MCI - patients $[t(1,163)=4.69, P<0.001$, Fig. 4B], with an estimated increase of $97.56 \pm 20.34 \mathrm{~cm}$ compared to MCI-. ACE-R score correlated with absolute distance errors across healthy control subjects and total MCI patients $[t(1,85)=2.89, P<0.01]$ and across $\mathrm{MCI}+$ and MCI- groups $[t(1,26)=4.01, P<0.01]$, with lower ACE$\mathrm{R}$ scores being associated with greater distance errors.

To assess whether tau and amyloid- $\beta$ contributed toward absolute distance error a mixed effect model was performed with both CSF measures z-scored, controlling for years in education, sex and age. It was found that the model explained $64 \%$ of the variance $\left(\mathrm{R}^{2}\right)$ with CSF biomarkers being significant predictors of absolute distance error. Absolute distance error was positively correlated with CSF total tau $[t(1,25)=2.18, P<0.05]$ and negatively associated with CSF amyloid- $\beta \quad[t(1,25)=-4.39$, $P<0.001$; Supplementary Fig. 2].

Neither sex, years in education or age were predictive of absolute distance error $(P>0.05)$. An interaction between CSF tau and CSF amyloid- $\beta$ was also examined but this addition neither improved model fit, as indicated by ratio likelihood testing, or was significant, and thus was not used in the final analysis.

Similar main effects of diagnosis were observed in proportional linear errors between healthy control subjects and total MCI group $[t(1,95)=2.27, P<0.05]$, as well as between $\mathrm{MCI}+$ and MCI- groups $[t(1,87)=3.09$, $P<0.001$ ] , but were not observed in proportional angular errors $(P>0.05$ across both groups; see Supplementary material). No other fixed effect was associated with any of the outcome measures of the path integration task.

\section{Effect of return condition on path integration}

No main effects of return condition was observed on absolute distance error between healthy control and total MCI groups $[F(2,1318)=0.86, P>0.05$; Fig. 5] or between $\mathrm{MCI}+$ and MCI- groups $[F(2,384)=0.56$, $P>0.05]$. Interaction terms between return condition and participant grouping were also included in the analyses to examine the differential influence of return conditions on path integration performance for $\mathrm{MCI}+$ (compared to MCI-) and MCI as a whole (compared to healthy control
A

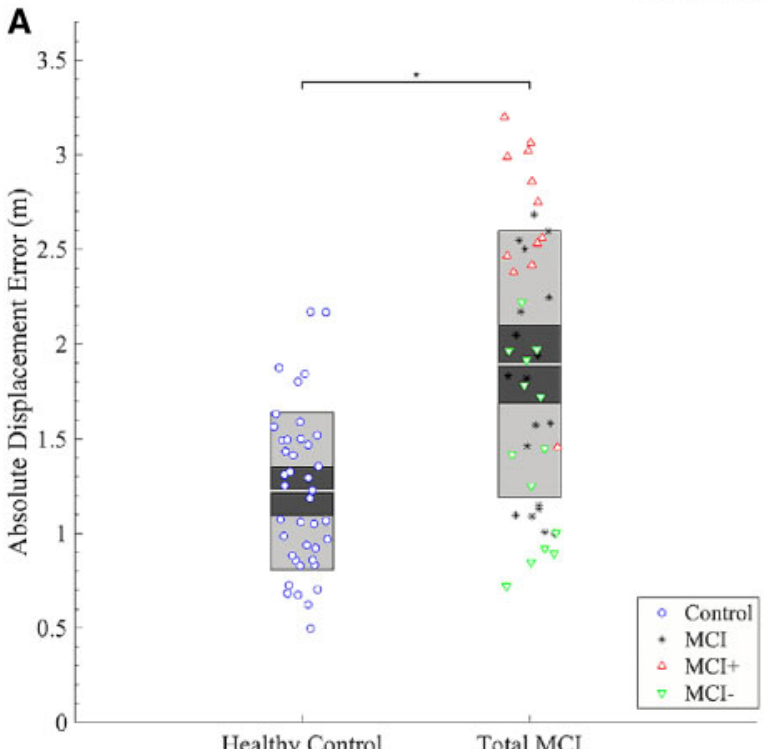

\section{Absolute Distance Error}

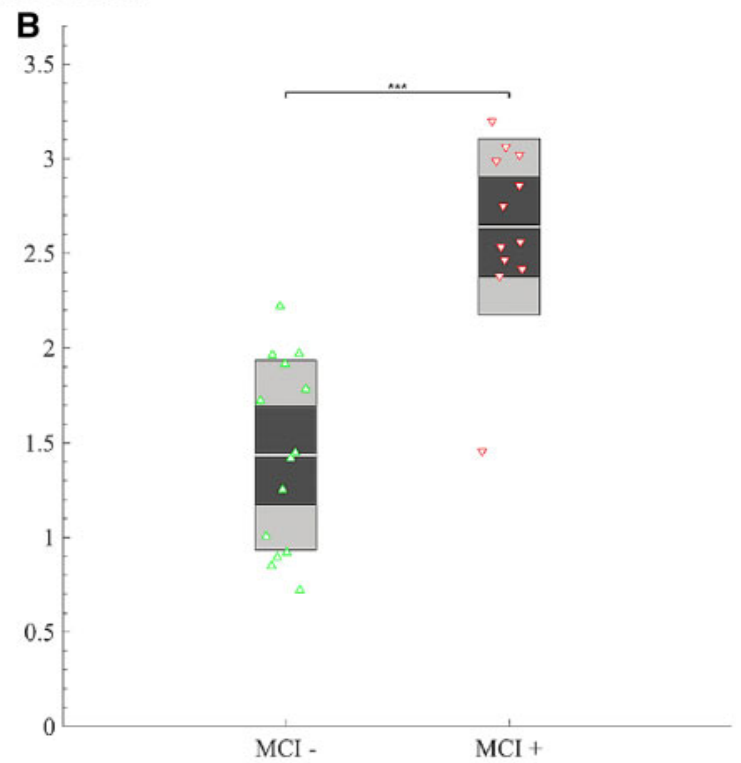

Figure 4 Graph summarizing the between group differences in path integration performance. Absolute distance error (Euclidean distance) error in metres ( $\mathbf{A}$ and $\mathbf{B})$. (A) Group comparison between healthy controls and total $\mathrm{MCl}$ and (B) between $\mathrm{MCl}-$ and $\mathrm{MCl}+$. Each marker represents the mean performance across trials of each individual: blue circles $=$ healthy control subjects; black asterisks $=\mathrm{MCl}$ without biomarkers; red triangles $=\mathrm{MCl}+$; green inverted triangles $=\mathrm{MCl}-$; central grey line $=$ mean; dark grey inner box $=95 \% \mathrm{Cls}$; light grey outer box $=$ I standard deviation. $* P<0.05$, $* * * P<0.001$. 


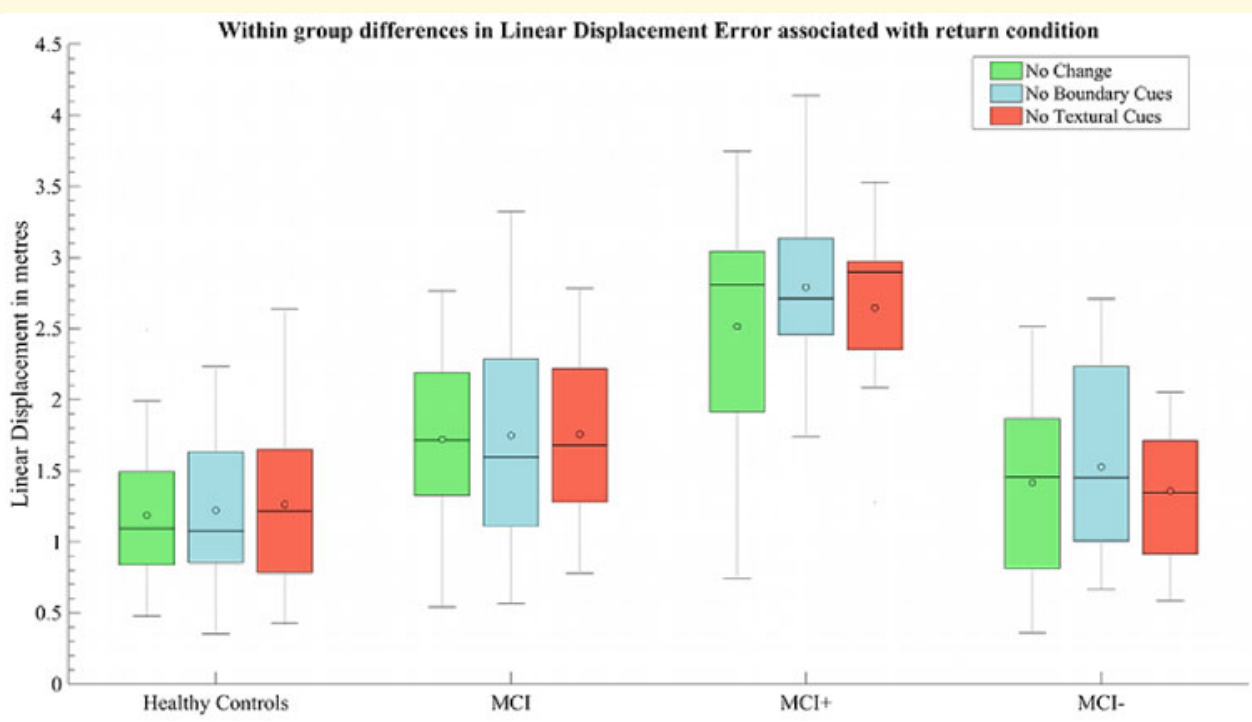

Figure 5 The effect of return condition within participant groups. The effect of return condition on absolute distance error averaged per participant in each group. Return conditions: green = no environmental change; blue = removal of distal boundary cues; red = removal of surface detail; open circle $=$ mean; black line $=$ median .

subjects). However, no interaction was observed between healthy control and total MCI groups $[F(2,1325)=0.83$, $P>0.05]$ or between $\mathrm{MCI}+$ and $\mathrm{MCI}-$ groups $[F(2,386)=0.55, P>0.05]$.

For proportional angular errors, a trend toward an interaction between biomarker status $(\mathrm{MCI}+$ versus $\mathrm{MCI}-$ ) and return conditions $\mathrm{B}$ and $\mathrm{C}$ was observed $[F(2,398)=2.93, P<0.05$; Supplementary Fig. $5 \mathrm{~A}]$, but this did not survive Bonferroni correction. No other main effect of return condition or interaction with diagnostic status was observed for proportional outcome measures $(P>0.05$, see Supplementary material).

\section{Group differences in MRI volumetry and association with path integration performance}

Group-level analyses adjusted for age, sex and years in education, revealed reduced region of interest volumetry (PCC, hippocampus, EC, alEC and pmEC) in the total MCI group compared to healthy control subjects $(P<0.05)$, and in the $\mathrm{MCI}+$ group compared to the MCI- group $(P<0.05)$. However, across healthy control subjects and total MCI, only hippocampal $[F(1,66)=13.32$, $P<0.001], \quad$ EC $\quad[F(1,66)=33.14, \quad P<0.001], \quad$ alEC $[\mathrm{F}(1,66)=21.87, P<0.001]$ and $\operatorname{pmEC}[F(1,66)=12.16$, $P<0.001]$ volumes survived the Bonferroni adjusted $\alpha$ of 0.005 . No volumetric difference of the isthmus was observed across all participants or $\mathrm{MCI}+$ and $\mathrm{MCI}-$ groups $(P>0.05$, see Supplementary Table 2 for full results).

Associations between regions of interest and path integration performance were assessed across total MCI and healthy control groups (Fig 6, purple line) and across $\mathrm{MCI}+$ and MCI- groups (Fig. 6, grey line), adjusting for age, sex, years in education and average path integration performance per participant group. Significant negative associations, surviving the Bonferroni adjusted $\alpha$ of 0.005 , were observed across all participants between absolute distance errors and both total EC $[F(1,64)=9.60, P<0.005$; Fig. 6A] and pmEC $[F(1,64)=9.73, P<0.005$; Fig. 6C] volumes, each with an $\mathrm{R}^{2}$ of 0.38 . Across healthy control and total MCI groups, associations between absolute distance error, alEC $(P=0.04$, Fig. 6B) and hippocampal $(P=0.03$, Fig. $6 \mathrm{D})$ volumes did not survive Bonferroni correction. Similarly, for $\mathrm{MCI}+$ and $\mathrm{MCI}-$ group comparisons, the association between hippocampal volume and absolute distance error (Fig. 6D, $P=0.02$ ) did not survive multiple comparison correction. Neither posterior cingulate nor isthmus cingulate volumes were significant predictors of absolute distance error across all participants (PCC: $t=1.95, P>0.05 ;$ isthmus cingulate cortex: $t=0.35$, $P>0.05)$ or MCI + and MCI- (PCC: $t=1.47, P>0.05$; isthmus cingulate cortex: $t=0.19, P>0.05$ ).

Additional analyses used multiple linear regression to examine the strength of EC and pmEC associations with absolute distance error whilst controlling for both hippocampal and alEC volumes across all participants. EC $[F(1,63)=6.09, P<0.05)$ and $\operatorname{pmEC}[F(1,63)=6.14, P<$ $0.03]$ models were significant, both EC $(t=2.20, P<0.05)$ and $\operatorname{pmEC}(t=2.23, P<0.05)$ volumes were significant predictors of absolute displacement error but neither survive multiple comparison corrections. To examine further the neural correlates of path integration performance, a hypothesis-free backward stepwise regression was performed, with absolute distance error as the response 


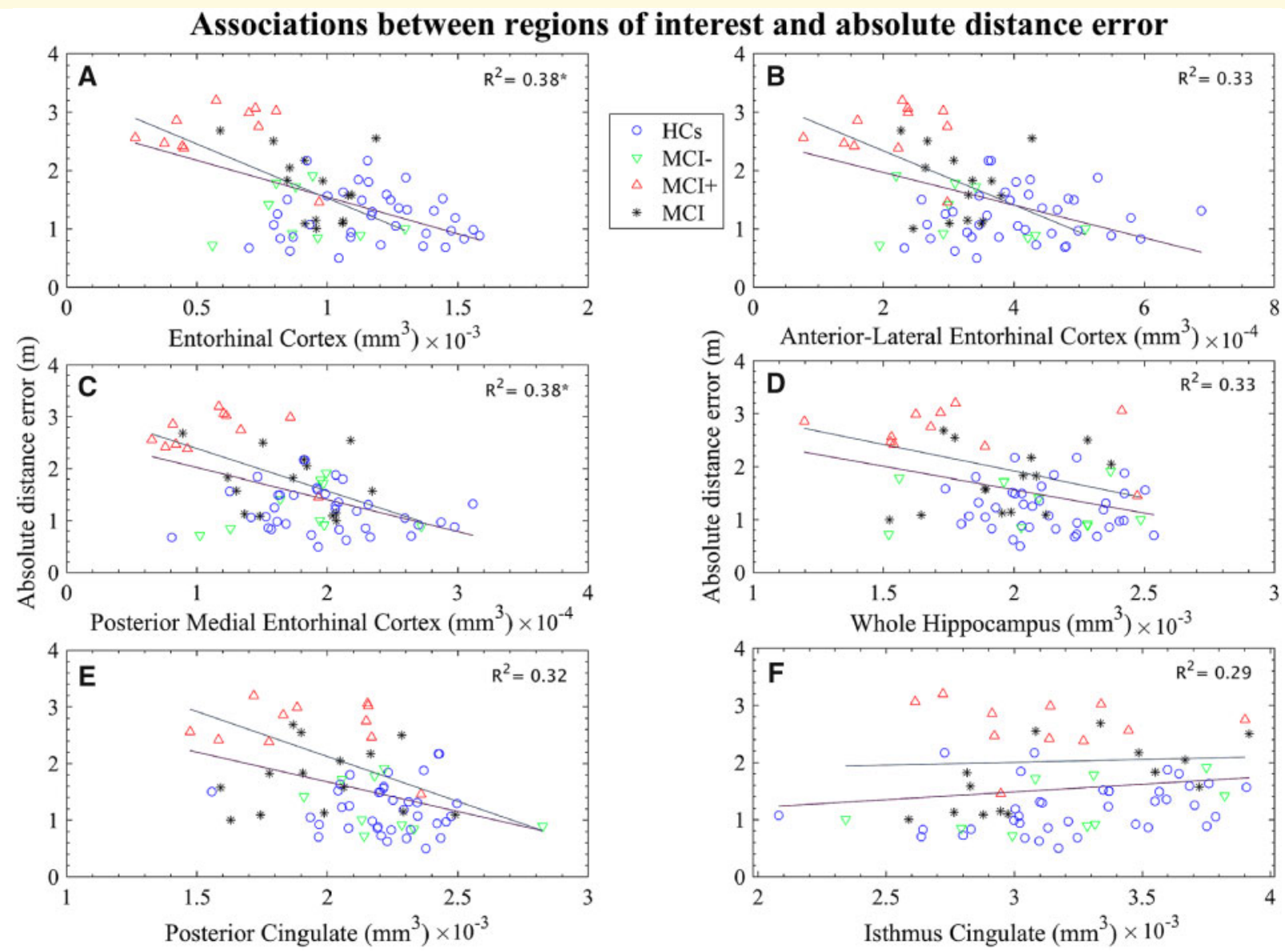

Figure 6 Scatterplots of path integration performance and region of interest volumetry. The relationship absolute distance error and regions of interest including; EC (A), alEC (B), pmEC (C), whole hippocampus (D), PCC (E) and isthus of the cingulate cortex (F) was assessed. Least square lines are group specific: grey $=$ across $\mathrm{MCl}+$ and $\mathrm{MCl}-$; purple $=$ all participants), $* P<0.005$ (Bonferroni adjusted $\alpha$ ).

variable and regions of interest from the Desikan-KillianyTourville atlas (Klein and Tourville, 2012, averaged across hemispheres and normalized to intracranial volume) as predictor variables, along with patient status, age, sex and years in education. Predictor variable inclusion was determined by the Akaike information criterion and the final model's predictor variables were refined by examination of variance inflation factors in an attempt to minimize collinearity. Finally, false discovery rate with a stringent alpha of 0.01 was used to control for multiple comparisons. The final model was significant $[F(34,36)=5.79, P<0.001]$, explaining $86.51 \%\left(\mathrm{R}^{2}\right)$ of the variance in absolute distance error. Following control for multiple comparisons, 20 brain volumes significantly contributed to the final model and these are summarized in Supplementary Table 3. However, stepwise regression can be affected by collinearity and as such the interpretation of these additional region of interest analyses needs to be made with caution (Thompson, 1995).

Lastly, given the demonstrated high accuracy of the 4MT in differentiating MCI due to underlying Alzheimer's disease (Moodley et al., 2015) and its predictive subsequent conversion to dementia (Wood et al., 2016), an ANCOVA adjusted for age, sex, years in education and average group performance was used to examine the relationship between 4MT scores and both the pmEC and hippocampus across all participants and $\mathrm{MCI}+$ and $\mathrm{MCI}-$. Across all participants, the model was significant for both hippocampal $[F(1,64)=6.54, \quad P<0.001] \quad$ and $\quad$ pmEC volumes $[F(1,64)=5.63, P<0.001]$; however, neither hippocampal $(t=1.92, P>0.05)$ or $\operatorname{pmEC}(t=0.73, P>0.05)$ volumes were significant predictors of 4MT score. Across $\mathrm{MCI}+$ and $\mathrm{MCI}-$ neither hippocampus $[F(1,14)=1.36$, $P<0.05]$ or pmEC models were significant $[F(1,14)=1.37, P>0.05]$.

\section{Receiver operating curves curves and classification accuracy}

AUC, sensitivity and specificity were estimated using k-fold cross-validation $(\mathrm{k}=10)$, adjusted for age, sex and years in education. For the classification of total MCI patients from healthy control subjects, absolute distance error was associated with an AUC of 0.82 [Fig. 7A; 95\% confidence 
intervals $(\mathrm{CI})=0.71-0.89]$, with an error $\geqslant 157 \mathrm{~cm}$ yielding a sensitivity of 0.84 and specificity of 0.68 . By comparison, the ACE-R was associated with an AUC $=0.86(\mathrm{CI}=0.79$ 0.94), TMT-B $\quad(\mathrm{AUC}=0.79, \quad \mathrm{CI}=0.68-0.87), \quad 4 \mathrm{MT}$ (AUC $=0.73, \mathrm{CI}=0.6-0.83)$ and the delayed conditions of FCSRT (AUC $=0.73, \quad \mathrm{CI}=0.61-0.85)$ and ReyOsterrieth Figure Recall Test (AUC $=0.72, \mathrm{CI}=0.60-0.83$ ).

Classification accuracy of MCI + from MCI- using absolute distance error was very high, with an AUC of 0.90 (Fig 7B; $\mathrm{CI}=0.59-1$ ), and errors $\geqslant 196 \mathrm{~cm}$ yielding a sensitivity and specificity of 0.92 for both. This AUC was considerably higher than that of the comparator reference cognitive tests: ACE-R (AUC $=0.53, \mathrm{CI}=0.24-0.73$ ), TMT-B $(\mathrm{AUC}=0.57, \mathrm{CI}=0.22-0.69), 4 \mathrm{MT}(\mathrm{AUC}=0.56$, $\mathrm{CI}=0.22-0.72)$ and the delayed conditions of FCSRT (AUC $=0.57, \mathrm{CI}=0.22-0.68)$ and Rey-Osterrieth Figure Recall Test (AUC $=0.55, \mathrm{CI}=0.22-0.68)$, indicating a markedly superior ability of the path integration test to differentiate $\mathrm{MCI}+$ from $\mathrm{MCI}-$.

\section{Discussion}

This study demonstrated that performance on a novel immersive virtual reality path integration paradigm, based on the central role of the entorhinal cortex in navigation, was impaired in MCI patients compared to healthy controls. In keeping with the study hypothesis that a navigation task based upon theories of EC function can differentiate MCI patients at increased risk of developing dementia, we found that Alzheimer's disease biomarker-positive patients drove the difference in navigation accuracy between MCI patients and controls. Consistent with the postulated role of the EC in navigation, and the specific role of the pmEC subdivision in spatial processing, larger path integration performance errors were associated with smaller total EC and pmEC subdivision volumes across all participants. Finally, and of high relevance for potential diagnostic usage, path integration performance differentiated MCI biomarker-positive patients, i.e. those with prodromal Alzheimer's disease, from biomarker-negative patients with markedly higher sensitivity and specificity than a battery of 'gold standard' cognitive tests used in clinical and research practice.

The navigational impairments observed in MCI patients is in line with previous navigation research (Hort et al., 2007; Laczó et al., 2014; Peter et al., 2018) and with the sparse literature on real-space path integration in MCI and Alzheimer's disease (Mokrisova et al., 2016). Significantly larger absolute distance errors were observed in MCI+ than in MCI-, with near-total separation of these two groups on this primary outcome measure, with the latter group exhibiting comparable performance to healthy control subjects. Additional analyses revealed that both CSF total tau and CSF amyloid- $\beta$ were highly predictive of

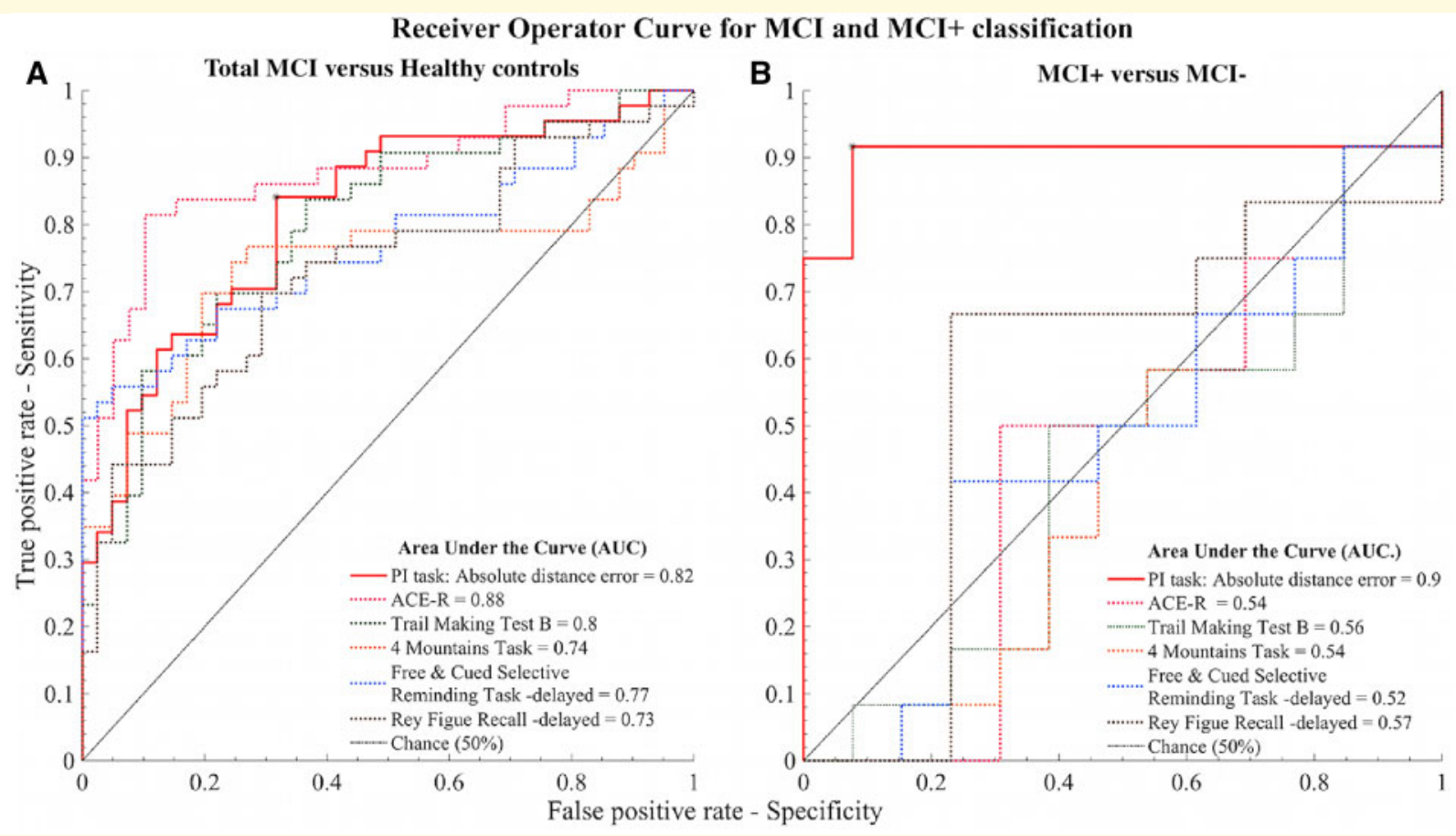

Figure 7 ROC plot. Accuracy of path integration task performance for classifying (A) total $\mathrm{MCl}$ from healthy control subjects and $(\mathbf{B}) \mathrm{MCl}+$ from $\mathrm{MCl}$ - patients. Path integration performance is represented by absolute distance error (solid red line). Classification of reference cognitive tests is represented by dashed lines for comparison. ACE-R (grey), Trail Making Test B (green), 4MT (yellow), Free and Cued Selective Reminding Test - delayed free recall (blue) and Rey Figure Recall - delayed recall (purple). Asterisk indicates optimal operating point for absolute distance error. 
absolute distance error, independent of age, sex and years in education, supporting the notion that path integration deficits are related to Alzheimer's disease molecular pathology. Collectively, these data suggest that navigational deficits are relatively specific to Alzheimer's disease and unrelated to deficits in other cognitive domains-such as attention or episodic memory-that might underlie the symptomatology of MCI- patients. Secondary outcome measures suggested that MCI + patients are specifically impaired in distance estimation, as evidenced by reduced proportional linear errors, in line with previous research (Hort et al., 2007), and may relate to tau-related disruption of grid cell activity (Fu et al., 2017; Stangl et al., 2018), given the role of grid cells in computing a distance metric of an environment (Bush et al., 2015) as part of path integration (McNaughton et al., 2006).

No group differences in performance errors were observed in response to the removal of boundary or surface detail cues. In the MCI + group, a trend toward increased proportional angular errors in response to the removal of boundary $(P=0.02)$ and textural $(P=0.08)$ cues was observed, but this did not survive multiple comparison correction. Given that this effect did not reach corrected statistical significance, any inferences need to be made with caution. Nonetheless it is worth noting that this trend is consistent with previous research that reported heightened increased reliance on landmark cues (Kalová et al., 2005) and heightened rotational deficits in response to the disruption of optic flow (Kavcic et al., 2006; Mapstone et al., 2008).

Decreased volume was observed in the MCI group compared to controls in regions of interest chosen for their role in path integration (total EC; including partial pmEC and alEC subdivisions, hippocampus, isthmus and posterior cingulate cortex), although only significant differences in hippocampal, total EC, pmEC and alEC volumes survived correction for planned comparisons. In contrast to previous research (Dickerson and Wolk, 2013; Long et al., 2018) no difference in region of interest volumetry across $\mathrm{MCI}+$ and MCI- patients survived correction, though this observation may be influenced by the sample sizes of these two groups. However, consistent with our hypothesis, total EC and partial pmEC subdivision volumes were negatively associated with absolute distance errors across all participants, contrasting with the lack of association between this behavioural measure and alEC, hippocampal, PCC and isthmus volume. Additional analyses demonstrate that both EC and partial pmEC volumes are better predictors of absolute distance errors than either hippocampal or alEC volumes. These findings reinforce previous work suggesting that the EC is critically involved in path integration, and that path integration is more dependent on the EC, and specifically the pmEC subdivision, than on the hippocampus or retrosplenial cortex (Shrager et al., 2008; Kim et al., 2013). To our knowledge, this is the first demonstration that reduced pmEC volumes are associated with impaired path integration in humans, and is consistent with the analogous role of the rodent $\mathrm{mEC}$ in path integration (McNaughton et al., 2006; Knierim et al., 2014). These findings complement previous research that demonstrates a relationship between the structure and function of the alEC in ageing and individuals at higher risk of Alzheimer's disease (Olsen et al., 2017; Berron et al., 2018) and sheds further light on the functional differentiation of the EC (Maass et al., 2015).

Significant negative associations were observed between absolute distance errors and both total EC and pmEC volumes, but not hippocampal volume. While these findings may support the role of the EC, and specifically pmEC, in path integration above and beyond the hippocampus, this interpretation must be applied with caution given that the significant structure-function association was only observed across all participants and not solely within the MCI+ group predicted to have EC degeneration. Future studies with increased sample size will be needed to explore this further.

Path integration performance differentiated the total MCI patient group from healthy control subjects with moderate classification accuracy (AUC 0.82), reflecting the large variance in performance within the former group. By comparison, path integration performance was highly sensitive and specific for prodromal Alzheimer's disease, classifying this group with an accuracy (AUC 0.90) that was markedly higher than that of reference cognitive tests of episodic memory, attention and processing speed widely used to diagnose prodromal Alzheimer's disease and as outcome measures in clinical trial.

This work contributes to the growing body of evidence that spatial behavioural tests may have added value, above and beyond traditional cognitive tests, in detecting pre-dementia Alzheimer's disease (Moodley et al., 2015; Allison et al., 2016; Coughlan et al., 2018; Ritchie et al., 2018). Furthermore, it demonstrates the potential added diagnostic value of a test based around theories of EC function. While this study focused on navigation, given knowledge of the underlying neural basis and the associated translational benefits of such an approach, there is evidence that the transentorhinal and alEC may be affected earlier than the pmEC by the spread of tau (Braak and Braak, 1991; Khan et al., 2014). As such, ongoing work involves extension of VR testing to encompass paradigms relating to object memory, reflecting transentorhinal and alEC function (Deshmukh and Knierim, 2011; Olsen et al., 2017; Berron et al., 2018).

One possible pathological explanation for these data is that impaired path integration in patients with prodromal Alzheimer's disease is due to spread of tau from the transentorhinal cortex to the pmEC and hippocampus and/or accumulation of amyloid- $\beta$ pathology in the retrosplenial cortex. The notion that the behavioural impairments are related to Alzheimer's disease pathology is reinforced by the observation of a significant association between path integration performance and both CSF total tau and CSF amyloid- $\beta$. The notion that the behavioural impairments are related to Alzheimer's disease pathology is reinforced 
by the observation of a significant association between path integration performance and both CSF total tau and CSF amyloid- $\beta$. Given converging data from studies on the initial cortical distribution of Alzheimer's disease molecular pathology (Braak and Braak, 1991; Bejanin et al., 2017; Whitwell et al., 2018), with tau deposition in the EC and amyloid- $\beta$ in the retrosplenial cortex, the path integration deficits observed here in prodromal Alzheimer's disease may relate to a combination of tau and amyloid- $\beta$-related dysfunction in the EC and retrosplenial cortex, respectively. Future studies using amyloid- and tau-PET will investigate these potential associations between molecular pathology and navigational behaviour in the early stages of Alzheimer's disease further, and the relative contribution of amyloid- $\beta$ and tau to the observed behavioural deficits.

This study has limitations. The sample size of both $\mathrm{MCI}+$ and MCI- groups was relatively small, and these results therefore need to be considered initial findings that require replication in larger scale studies. Another limitation concerns the test space available with the commercial iVR hardware. The use of a larger space, which will be possible with next generation iVR, would likely result in the (i) exclusion of fewer trials; (ii) evaluation of proportional linear errors that is not skewed towards an undershoot; and (iii) the compounding of vector computation errors (angular and linear estimates) that would likely culminate in larger between group performance differences. Finally, the lack of an anatomical mask for automated measurement of the retrosplenial cortex, necessitating the use of proximal anatomical measures (PCC and isthmus cingulate cortex volumes), limits the specificity of analysis of the possible contribution of retrosplenial cortex dysfunction to the path integration impairment in prodromal Alzheimer's disease.

In conclusion, this study demonstrates that performance on an EC-based iVR path integration task is sensitive and specific for prodromal Alzheimer's disease, with greater classification accuracy than that of a battery of current 'gold standard' cognitive tests. Given that this test is based on understanding of EC grid cell activity, these findings have implications not just for early diagnosis but also for translational Alzheimer's disease research aimed at understanding mechanistic links between impaired cell activity and behaviour in Alzheimer's disease. The task used in this study, combined with analogous navigation tasks in animal models of Alzheimer's disease, would help address the need for outcome measures capable of comparing treatment effects across preclinical and clinical phases of future treatment trials aimed at delaying or preventing the onset of dementia.

\section{Acknowledgements}

The authors thank Daniel Bush for his comments on the manuscript and Zoe Adler for her help recruiting and testing patients.

\section{Funding}

This work was supported by the Wellcome Trust Fellowship (202805/Z/16/Z), the European Research Council grant (ERC-2015-Adg, 694779) to N.B. A.C. is sponsored by UCL's Institute for Communications and Connected Systems. D.H. is supported by a UK Medical Research Council/Raymond and Beverly Sackler PhD studentship along with pump-priming support from Alzheimer's Research UK. R.H. is supported by MRC Programme Grant SUAG/010 RG91365. D.C. is funded by the Wellcome Trust, Isaac Newton Trust and the Cambridge NIHR Biomedical Research Centre.

\section{Competing interests}

The authors report no competing interests.

\section{Supplementary material}

Supplementary material is available at Brain online.

\section{References}

Adamo DE, Briceño EM, Sindone JA, Alexander NB, Moffat SD, May $\mathrm{M}$, et al. Age differences in virtual environment and real world path integration. Front Aging Neurosci 2012; 4: 26.

Ahmed Z, Cooper J, Murray TK, Garn K, McNaughton E, Clarke H, et al. A novel in vivo model of tau propagation with rapid and progressive neurofibrillary tangle pathology: The pattern of spread is determined by connectivity, not proximity. Acta Neuropathol 2014; 127: 667-83.

Allen K, Gil M, Resnik E, Toader O, Seeburg P, Monyer H. Impaired path integration and grid cell spatial periodicity in mice lacking GluA1-containing AMPA receptors. J Neurosci 2014; 34: 6245-59.

Allison SL, Fagan AM, Morris JC, Head D. Spatial navigation in preclinical Alzheimer's disease. J Alzheimer's Dis 2016; 52: 77-90.

Association WM. World medical association declaration of Helsinki: ethical principles for medical research involving human subjects. JAMA 2013; 310: 2191-4.

Bejanin A, Schonhaut DR, La Joie R, Kramer JH, Baker SL, Sosa N, et al. Tau pathology and neurodegeneration contribute to cognitive impairment in Alzheimer's disease. Brain 2017; 140: 3286-300.

Berron D, Neumann K, Maass A, Schutze H, Fliessbach K, Kiven V, et al. Age-related functional changes in domain-specific medial temporal lobe pathways. Neurobiol Aging 2018; 65: 86-97.

Berron D, Vieweg P, Hochkeppler A, Pluta JB, Ding SL, Maass A, et al. A protocol for manual segmentation of medial temporal lobe subregions in 7 Tesla MRI. NeuroImage Clin 2017; 15: 466-82.

Bohbot VD, Copara MS, Gotman J, Ekstrom AD. Low-frequency theta oscillations in the human hippocampus during real-world and virtual navigation. Nat Commun 2017; 8: 14415.

Bowie CR, Harvey PD. Administration and interpretation of the Trail Making Test. Nat Protoc 2006; 1: 2277-81.

Braak H, Braak E. Neuropathological stageing of Alzheimer-related changes. Acta Neuropathol 1991; 82: 239-59.

Braak H, Del Tredici K. The preclinical phase of the pathological process underlying sporadic Alzheimer's disease. Brain 2015; 138 2814-33. 
Burgess N. Grid cells and theta as oscillatory interference: theory and predictions. Hippocampus 2008; 18: 1157-74.

Burgess N, Spiers HJ, Paleologou E. Orientational manoeuvres in the dark: dissociating allocentric and egocentric influences on spatial memory. Cognition 2004; 94: 149-66.

Buschke H. Cued recall in Amnesia. J Clin Neuropsychol 1984; 6: 433-40.

Bush D, Barry C, Burgess N, Bush D, Barry C, Manson D, et al. Using grid cells for navigation. Neuron 2015; 87: 507-20.

Campbell MG, Ocko SA, Mallory CS, Low IIC, Ganguli S, Giocomo LM. Principles governing the integration of landmark and selfmotion cues in entorhinal cortical codes for navigation. Nat Neurosci 2018; 21: 1096-106.

Chadwick MJ, Jolly AEJ, Amos DP, Hassabis D, Spiers HJ. Report a goal direction signal in the human entorhinal/subicular region. Curr Biol 2015; 25: 87-92.

Chrastil ER, Sherrill KR, Hasselmo ME, Stern CE. There and back again: hippocampus and retrosplenial cortex track homing distance during human path integration. J Neurosci 2015; 35: 15442-52.

Conover WJ, Iman RL. Analysis of covariance using the rank transformation. Biometrics 1982; 38: 715-24.

Coughlan G, Laczó J, Hort J, Minihane AM, Hornberger M. Spatial navigation deficits - the overlooked cognitive fingerprint for incipient Alzheimer pathophysiology? Nat Rev Neurol 2018; 14: 496506.

de Calignon A, Polydoro M, Suárez-Calvet M, William C, Adamowicz $\mathrm{DH}$, Kopeikina KJ, et al. Propagation of tau pathology in a model of early Alzheimer's disease. Neuron 2012; 73: 685-97.

Deshmukh SS, Knierim JJ. Representation of non-spatial and spatial information in the lateral entorhinal cortex. Front Behav Neurosci 2011; 5: 69 .

Dickerson BC, Wolk DA. Biomarker-based prediction of progression in MCI: Comparison of $\mathrm{AD}$ signature and hippocampal volume with spinal fluid amyloid- $\beta$ and tau. Front Aging Neurosci 2013; 5: $1-9$.

Diehl GW, Hon OJ, Leutgeb S, Leutgeb JK. Grid and nongrid cells in medial entorhinal cortex represent spatial location and environmental features with complementary coding schemes. Neuron 2017; 94: 83-92.e6.

Distler HK, Van Veen HAHC, Braun SJ, Heinz W, Franz MO, Biilthoff $\mathrm{HH}$. Navigation in real and virtual environments: judging orientation and distance in a large-scale landscape. In: Göbel M, Landauer J, Lang U, Wapler M, editors. Virtual Environments'98. Proceedings of the Eurographics Work, Stuttgart. Vienna: Springer; 1998. p. 124-33.

Doeller CF, King JA, Burgess N. Parallel striatal and hippocampal systems for landmarks and boundaries in spatial memory. Proc Natl Acad Sci USA 2008; 105: 5915-20.

Etienne AS, Jeffery KJ. Path integration in mammals. Hippocampus 2004; 14: 180-192.

Fischl B, Salat DH, Busa E, Albert M, Dieterich M, Haselgrove C, et al. Whole brain segmentation: automated labeling of neuroanatomical structures in the human brain. Neuron 2002; 33: 341-55.

Fu H, Rodriguez GA, Herman M, Emrani S, Nahmani E, Barrett G, et al. Tau pathology induces excitatory neuron loss, grid cell dysfunction, and spatial memory deficits reminiscent of early Alzheimer's disease. Neuron 2017; 93: 533-41.e5.

Fyhn M, Hafting T, Treves A, Moser M-B, Moser EI. Hippocampal remapping and grid realignment in entorhinal cortex. Nature 2007; 446: $190-4$.

Gomez-Isla T, Hollister R, West H, Mui S, Growdon JH, Petersen RC, et al. Neuronal loss correlates with but exceeds neurofibrillary tangles in Alzheimer's disease. Ann Neurol 1997; 41: 17-24.

Hafting T, Fyhn M, Molden S, Moser MB, Moser EI. Microstructure of a spatial map in the entorhinal cortex. Nature 2005; 436: 801-6.

Harris MA, Wolbers T. Ageing effects on path integration and landmark navigation. Hippocampus 2012; 22: 1770-80.
Hartley T, Bird CM, Chan D, Cipolotti L, Husain M, Vargha-Khadem $\mathrm{F}$, et al. The hippocampus is required for short-term topographical memory in humans. Hippocampus 2007; 17: 34-48.

Hawkins DM, Basak SC, Mills D. Assessing model fit by cross-validation. J Chem Inf Comput Sci 2003; 43: 579-86.

Hort J, Laczo J, Vyhnalek M, Bojar M, Bures J, Vlcek K. Spatial navigation deficit in amnestic mild cognitive impairment. Proc Natl Acad Sci 2007; 104: 4042-7.

Howard LR, Javadi AH, Yu Y, Mill RD, Morrison LC, Knight R, et al. Article the hippocampus and entorhinal cortex encode the path and euclidean distances to goals during navigation. Curr Biol 2014; 24: $1331-40$.

Iglesias JE, Augustinack JC, Nguyen K, Player CM, Player A, Wright $\mathrm{M}$, et al. A computational atlas of the hippocampal formation using ex vivo, ultra-high resolution MRI: Application to adaptive segmentation of in vivo MRI. Neuroimage 2015; 115: 117-37.

Jacob PY, Salas MG, Facchini J, Poucet B, Save E, Sargolini F. Medial entorhinal cortex and medial septum contribute to self- motionbased linear distance estimation. Brain Struct Funct 2017; 222: 2727-42.

Jacobs J, Kahana MJ, Ekstrom AD, Mollison MV, Fried I. A sense of direction in human entorhinal cortex. Proc Natl Acad Sci 2010; 107: 6487-92.

Jacobs J, Weidemann CT, Miller JF, Solway A, Burke JF, Wei XX, et al. Direct recordings of grid-like neuronal activity in human spatial navigation. Nat Neurosci 2013; 16: 1188-90.

Kalová E, Vlček K, Jarolímová E, Bureš J. Allothetic orientation and sequential ordering of places is impaired in early stages of Alzheimer's disease: corresponding results in real space tests and computer tests. Behav Brain Res 2005; 159: 175-86.

Kavcic V, Fernandez R, Logan D, Duffy CJ. Neurophysiological and perceptual correlates of navigational impairment in Alzheimer's disease. Brain 2006; 129: 736-46.

Kearns MJ, Warren WH, Duchon AP, Tarr MJ. Path integration from optic flow and body senses in a homing task. Perception 2002; 31: 349-74.

Khan UA, Liu L, Provenzano FA, Berman DE, Profaci CP, Sloan R, et al. Molecular drivers and cortical spread of lateral entorhinal cortex dysfunction in preclinical Alzheimer's disease. Nat Neurosci 2014; 17: 304-11.

Killian NJ, Jutras MJ, Buffalo EA. A map of visual space in the primate entorhinal cortex. Nature 2012; 491: 761-4.

Kim S, Sapiurka M, Clark RE, Squire LR. Contrasting effects on path integration after hippocampal damage in humans and rats. Proc Natl Acad Sci USA 2013; 110: 4732-7.

Klatzky RL, Loomis JM, Beall AC, Chance SS, Golledge RG. Spatial updating of self-position and orientation during real, imagined, and virtual locomotion. Psychol Sci 1998; 9: 293-8.

Klein A, Tourville J. 101 labeled brain images and a consistent human cortical labeling protocol. Front Neurosci 2012; 6: 171.

Knierim JJ, Neunuebel JP, Deshmukh SS. Functional correlates of the lateral and medial entorhinal cortex: objects, path integration and local - global reference frames. Philos Trans R Soc Lond B Biol Sci 2014; 369: 20130369.

Kunz L, Schroder TN, Lee H, Montag C, Lachmann B, Sariyska R, et al. Reduced grid-cell-like representations in adults at genetic risk for Alzheimer's disease. Science 2015; 350: 430-3.

Laczó J, Andel R, Vyhnalek M, Vlcek K, Nedelska Z, Matoska V, et al. APOE and spatial navigation in amnestic MCI: Results from a computer-based test. Neuropsychology 2014; 28: 676-84.

Lester AW, Moffat SD, Wiener JM, Barnes CA, Wolbers T. The aging navigational system. Neuron 2017; 95: 1019-35.

Long X, Jiang C, Zhang L. Morphological biomarker differentiating MCI converters from nonconverters: longitudinal evidence based on hemispheric asymmetry. Behav Neurol 2018; 2018: 1-9.

Maass A, Berron D, Libby L, Ranganath C, Düzel E. Functional subregions of the human entorhinal cortex. Elife 2015; 4: 1-20. 
Maguire EA, Burgess N, Donnett JG, Frackowiak RS, Frith CD, O'Keefe J. Knowing where and getting there: a human navigation network. Science 1998; 280: 921-4.

Mapstone M, Dickerson K, Duffy CJ. Distinct mechanisms of impairment in cognitive ageing and Alzheimer's disease. Brain 2008; 131(Pt 6): 1618-29.

McNaughton BL, Battaglia FP, Jensen O, Moser EI, Moser M-B. Path integration and the neural basis of the 'cognitive map'. Nat Rev Neurosci 2006; 7: 663-78.

Mehta D, Jackson R, Paul G, Shi J, Sabbagh M. Why do trials for Alzheimer's disease drugs keep failing? A discontinued drug perspective for 2010-2015. Expert Opin Investig Drugs 2017; 26: 735-9.

Mioshi E, Dawson K, Mitchell J, Arnold R, Hodges JR. The Addenbrooke's Cognitive Examination revised (ACE-R): a brief cognitive test battery for dementia screening. Int J Geriatr Psychiatry 2006; 21: 1078-85.

Mitchell AS, Czajkowski R, Zhang N, Jeffery K, Nelson AJD. Retrosplenial cortex and its role in spatial cognition. Brain Neurosci Adv 2018; 2: 2398212818757098.

Moen EL, Fricano-Kugler CJ, Luikart BW, O’Malley AJ. Analyzing clustered data: Why and how to account for multiple observations nested within a study participant? PLoS One 2016; 11: 1-17.

Mokrisova I, Laczo J, Andel R, Gazova I, Vyhnalek M, Nedelska Z, et al. Real-space path integration is impaired in Alzheimer's disease and mild cognitive impairment. Behav Brain Res 2016; 307: 150-8.

Moodley K, Minati L, Contarino V, Prioni S, Wood R, Cooper R, et al. Diagnostic differentiation of mild cognitive impairment due to Alzheimer's disease using a hippocampus-dependent test of spatial memory. Hippocampus 2015; 25: 939-51.

Moroney JT, Bagiella E, Desmond DW, Hachinski VC, Mölsä PK, Gustafson L, et al. Meta-analysis of the Hachinski Ischemic Score in pathologically verified dementias. Neurology 1997; 49: 1096105.

Morris JC. Clinical Dementia Rating: A reliable and valid diagnostic and staging measure for dementia of the Alzheimer type. In: International Psychogeriatrics. 1997. p. 173-176.

Mulder C, Verwey NA, van der Flier WM, Bouwman FH, Kok A, van Elk EJ, et al. Amyloid-beta(1-42), total tau, and phosphorylated tau as cerebrospinal fluid biomarkers for the diagnosis of Alzheimer disease. Clin Chem 2010; 56: 248-53.

Nardini M, Jones P, Bedford R, Braddick O. Development of cue integration in human navigation. Curr Biol 2008; 18: 689-93.

Naumann RK, Preston-Ferrer P, Brecht M, Burgalossi A. Structural modularity and grid activity in the medial entorhinal cortex. J Neurophysiol 2018; 119: 2129-44.

Naumann RK, Ray S, Prokop S, Las L, Heppner FL, Brecht M. Conserved size and periodicity of pyramidal patches in layer 2 of medial/caudal entorhinal cortex. J Comp Neurol 2016; 524: 783806.

Navarro Schröder T, Haak KV, Zaragoza Jimenez NI, Beckmann CF, Doeller CF. Functional topography of the human entorhinal cortex. Elife 2015; 4: 1-17.

Nelson HE. The National Adult Reading Test (NART): test manual. Wind. UK NFER-Nelson 1982; 124: 0-25.

O'Keefe J, Dostrovsky J. The hippocampus as a spatial map. Preliminary evidence from unit activity in the freely-moving rat. Brain Res 1971; 34: 171-175.

O'Keefe J, Nadel L. The hippocampus as a cognitive map. Oxford: Clarendon Press; 1978.

Ólafsdóttir HF, Barry C. Spatial cognition: Grid cell firing depends on self-motion cues. Curr Biol 2015; 25: R827-9.

Olsen RK, Yeung LK, Noly-Gandon A, D'Angelo MC, Kacollja A, Smith VM, et al. Human anterolateral entorhinal cortex volumes are associated with cognitive decline in aging prior to clinical diagnosis. Neurobiol Aging 2017; 57: 195-205.

Osterrieth PA. Le test de copie d'une figure complexe; contribution à l'étude de la perception et de la mémoire. Arch Psychol (Geneve) 1944; 30: 206-356.
Parron C, Save E. Evidence for entorhinal and parietal cortices involvement in path integration in the rat. Exp Brain Res 2004; 159: 349-59.

Peter J, Sandkamp R, Minkova L, Schumacher LV, Kaller CP, Abdulkadir A, et al. Real-world navigation in amnestic mild cognitive impairment: the relation to visuospatial memory and volume of hippocampal subregions. Neuropsychologia 2018; 109: 86-94.

Petersen RC. Mild cognitive impairment as a diagnostic entity. J Intern Med 2004; 256: 183-94.

Reagh ZM, Noche JA, Tustison NJ, Delisle D, Murray EA, Yassa MA. Functional imbalance of anterolateral entorhinal cortex and hippocampal dentate/CA3 underlies age-related object pattern separation deficits. Neuron 2018; 97: 1187-1198.e4.

Reagh ZM, Yassa MA. Object and spatial mnemonic interference differentially engage lateral and medial entorhinal cortex in humans. Proc Natl Acad Sci USA 2014; 111: E4264-73.

Ritchie K, Carrière I, Howett D, Su L, Hornberger M, O’Brien JT, et al. Allocentric and egocentric spatial processing in middle-aged adults at high risk of late-onset Alzheimer's disease: the PREVENT dementia study. J Alzheimer's Dis 2018; 65: 885-96.

Ryan JJ, Lopez SJ. Wechsler adult intelligence scale-III BT. In: Dorfman WI, Hersen M, editors. Understanding Psychological Assessment. Boston, MA: Springer US; 2001. p. 19-42.

Sargolini F, Fyhn M, Hafting T, McNaughton BL, Witter MP, Moser $\mathrm{M}-\mathrm{B}$, et al. Conjunctive representation of position, direction, and velocity in entorhinal cortex. Science 2006; 312: 758-62.

Shaw LM, Vanderstichele H, Knapik-Czajka M, Clark CM, Aisen PS, Petersen RC, et al. Cerebrospinal fluid biomarker signature in Alzheimer's disease neuroimaging initiative subjects. Ann Neurol 2009; 65: 403-13.

Shrager Y, Kirwan CB, Squire LR. Neural basis of the cognitive map: Path integration does not require hippocampus or entorhinal cortex. Proc Natl Acad Sci 2008; 105: 12034-8.

Sinai, Krebs, Darken W, Rp, Rowland, Mccarley J. Egocentric distance perception in a virutal environment using a perceptual matching task. Proc Hum Factors Ergonormics Soc 1999.

Solstad T, Boccara CN, Kropff E, Moser M-B, Moser EI. Representation of geometric borders in the entorhinal cortex. Science 2008; 322: 1865-8.

Spiers HJ, Maguire EA. A navigational guidance system in the human brain. Hippocampus 2007; 17: 618-26.

Stangl M, Achtzehn J, Huber K, Dietrich C, Tempelmann C, Wolbers $\mathrm{T}$, et al. Compromised grid-cell-like representations in old age as a key mechanism to explain age-related report compromised grid-celllike representations in old age as a key mechanism to explain agerelated navigational deficits. Curr Biol 2018; 28: 1108-15.e6.

Tennant SA, Fischer L, Garden DLF, Gerlei KZ, Martinez-Gonzalez C, McClure C, et al. Stellate cells in the medial entorhinal cortex are required for spatial learning. Cell Rep 2018; 22: 1313-24.

Thompson B. Stepwise regression and stepwise discriminant analysis need not apply here: a guidelines editorial. Educ Psychol Meas 1995; 55: 525-34.

Van Cauter T, Camon J, Alvernhe A, Elduayen C, Sargolini F, Save E. Distinct roles of medial and lateral entorhinal cortex in spatial cognition. Cereb Cortex 2013; 23: 451-9.

Vann SD, Aggleton JP, Maguire EA. What does the retrosplenial cortex do? Nat Rev Neurosci. 2009; 10: 792-802.

Whitwell JL, Graff-Radford J, Tosakulwong N, Weigand SD, Machulda M, Senjem ML, et al. [(18) F]AV-1451 clustering of entorhinal and cortical uptake in Alzheimer's disease. Ann Neurol 2018; 83: 248-57.

Winter SS, Mehlman ML, Clark BJ, Taube JS. Passive transport disrupts grid signals in the parahippocampal cortex. Curr Biol 2015; 25: 2493-502.

Wood RA, Moodley KK, Lever C, Minati L, Chan D. Allocentric spatial memory testing predicts conversion from mild cognitive impairment to dementia: an initial proof-of-concept study. Front Neurol 2016; 7: 215. 
Worsley CL, Recce M, Spiers HJ, Marley J, Polkey CE, Morris RG. Path integration following temporal lobectomy in humans. Neuropsychologia 2001; 39: 452-64.

Yartsev MM, Witter MP, Ulanovsky N. Grid cells without theta oscillations in the entorhinal cortex of bats. Nature 2011; 479: 103-7.

Yeung L-K, Olsen RK, Bild-Enkin HEP, D’Angelo MC, Kacollja A, McQuiggan DA, et al. Anterolateral entorhinal cortex volume predicted by altered intra-item configural processing. J Neurosci 2017; 37: 5527-38.
Yushkevich PA, Piven J, Hazlett HC, Smith RG, Ho S, Gee JC, et al. User-guided 3D active contour segmentation of anatomical structures: significantly improved efficiency and reliability. Neuroimage 2006; 31: 1116-28.

Zhao M, Warren WH. Environmental stability modulates the role of path integration in human navigation. Cognition 2015; 142: $96-$ 109. 